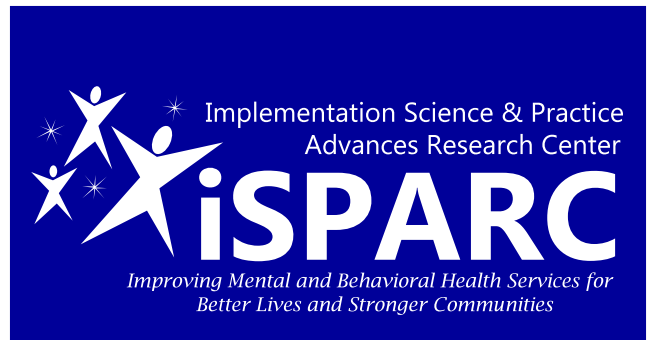

Tip Sheet

\title{
WAYS TO MIND YOUR MENTAL HEALTH IN CHALLENGING TIMES: TIPS FOR YOUTH
}
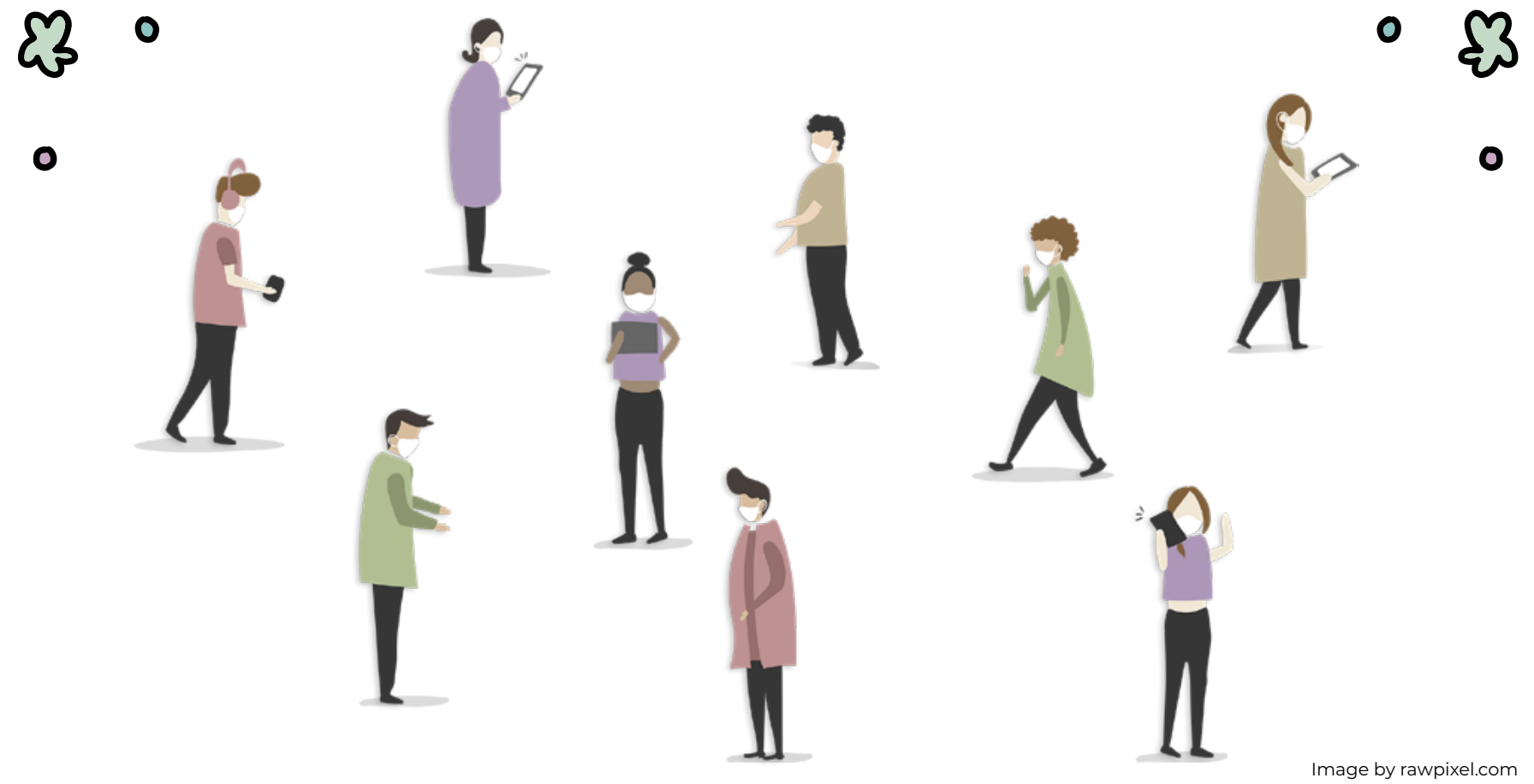

\section{CONNECT WITH OTHERS.}

In COVID times, it can be harder to stay in touch, but there are still safe ways to connect.

$\checkmark$ Meet a friend for an outdoor walk or a picnic.

$\checkmark$ Schedule a weekly video call with your closest friend.

$\checkmark$ Find a common interest and have "quality" time with those in your bubble. Ask someone you live with to teach you something they're good at, whether it's baking, playing an instrument or a video game.

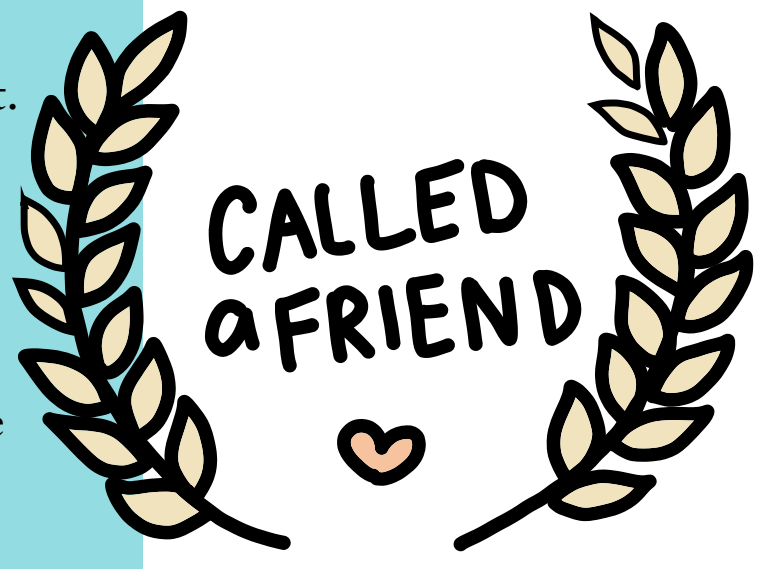

$\checkmark$ Set up a group Zoom, maybe include trivia about your favorite music or TV shows. 


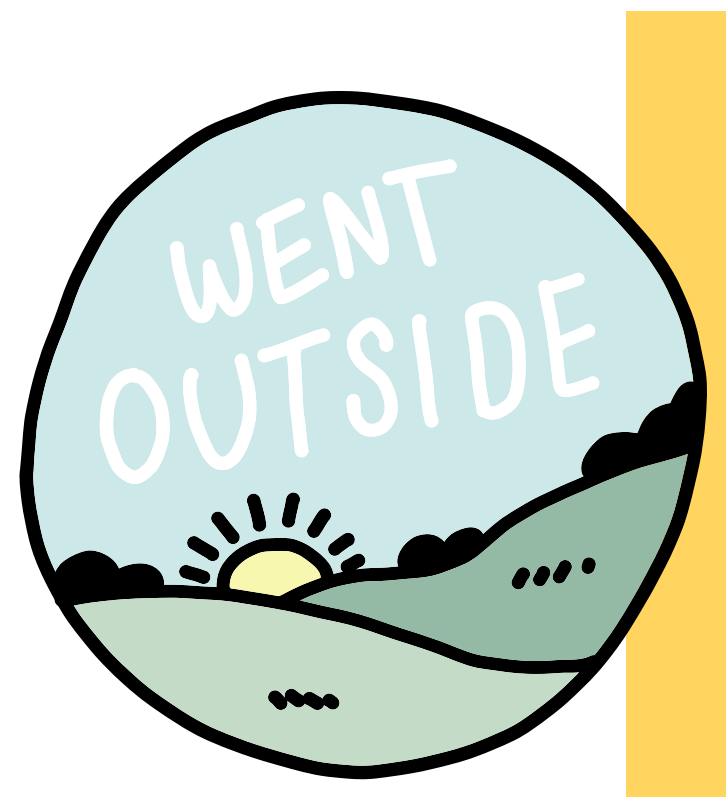

\section{GET MOVING!}

Sometimes when you're fed up or worn

out, the last thing you want to do is exercise. Finding one physical activity you like and doing it on a regular basis, can make a huge difference to how you feel.

$\checkmark$ Re-create dances from TikTok, music videos or YouTube dance tutorials: the faster the pace the better!

$\checkmark$ Take a walk. If you find walking boring, listen to music or a podcast as you go, or phone a friend.

$\checkmark$ Look for equipment-free workouts online. These will vary in length and difficulty so sample a few to find a good fit for you.

\section{HELP OTHERS.}

The pandemic has led to a lot of need in the community. Helping others can give you chances

to connect, get moving, and feel like you're making a difference, It can help you feel better. It might be a little harder to find ways to volunteer if you're under 18, but public libraries, schools, churches, and the American Red Cross are places youth and young adults can volunteer.

$\checkmark$ Vaccination centers are looking for volunteers to help keep sites efficient and safe and to provide transport for community members to get to their appointments. About one-in-ten households in the U.S. sometimes don't have enough to eat: check out local food banks or community pantries/fridges to see how you can help.

$\checkmark$ Choose a cause that's close to your heart, whether that's animal welfare, social justice, sports, mental health, or cancer prevention. Many organizations need remote volunteers to help fundraise, make phone calls or create content.

$\checkmark$ Formal volunteering isn't the only way to help others. Offer to do yard work, babysit, or pick up groceries for neighbors or relatives if you want to create an instant feel-good moment!

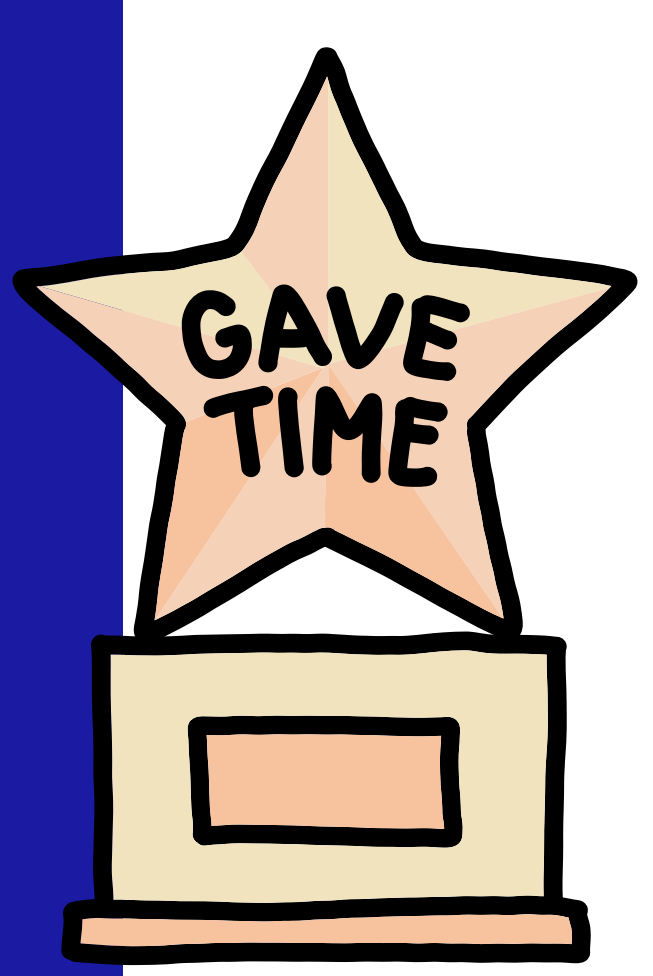




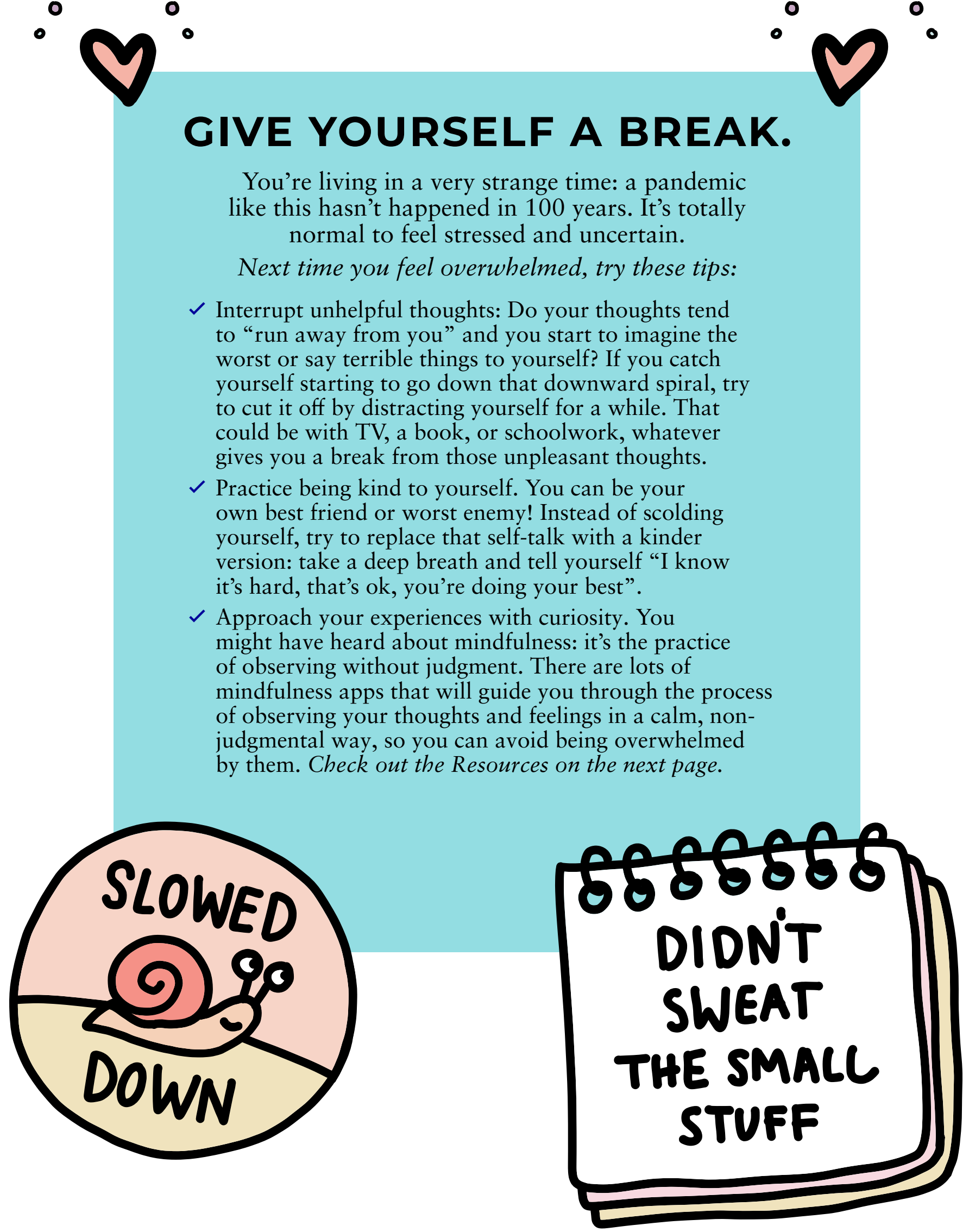




\section{KNOW WHEN TO SEEK HELP.}

Everyone can feel a little down or stressed from time to time but sometimes we need some extra support to guide us through a really tough time. There are support lines like the Samaritans or the Trevor

Project (see Resources) that you can reach out to anytime for a chat about what's going on, with someone who will just listen.

Sometimes, you might need a bit more help, for example if you:

$\checkmark$ Feel very low or very anxious more days than not, for a period of more than a couple of weeks.

$\checkmark$ Are harming your body, for example by cutting or burning it, eating too much or too little on purpose, over-exercising, drinking alcohol, or taking drugs.

$\checkmark$ Notice that your distress starts to disrupt activities you used to enjoy or be good at, like if feeling anxious prevents you from your usual hobbies or if you are falling behind in school.

$\checkmark$ Feel that your emotions, thoughts, and behaviors are often "out of control."

$\checkmark$ Start to wish you were dead or wish you could fall asleep and not wake up.

$\checkmark$ Have experienced a major life change or loss, like a death of a loved one, loss of a pet or service animal, move, illness, or family changes.

\section{RESOURCES}

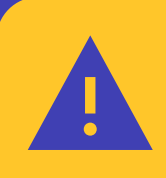

If any of these apply to you, consider speaking with a trusted adult and setting up an appointment with a school counselor, therapist, or primary care provider.

- Physical activity apps: Zombies, Run! app (\$5.99/month or $\$ 34.99 /$ year); Yoga for Beginners app (free); Nike Training Club app (free)

- Helping others: Volunteer Match (volunteermatch.org or teenlife.com/category/volunteer)

- Mindfulness apps: Smiling mind app (free), Healthy Minds Program app (free)

\section{HELPLINES}

- Samaritans - (call or text 877-870-4673 any time);

- Trevor Project - Saving Young LGBTQ Lives (call 1-866-488-7386 or text START to 678678 any time); National Suicide Prevention Lifeline (call 1-800-273-8255 any time) or visit thetrevorproject.org

- Crisis Text Line - Text "HOME" to 741741 to connect with a crisis counselor or visit crisistextline.org

This is a publication of the Implementation Science and Practice Advances Research Center (iSPARC) a Massachusetts Department of Mental Health Research Center of Excellence and The Learning and Working Transitions RRTC. The
contents of this tip sheet were supported in part with funding from the Massachusetts Department of Mental Health and under grants with funding from the National Institute on Disability, Independent Living, and Rehabilitation Research, (NIDILRR), United States Departments of Health and Human Services (NIDILRR grant number 9ORTEMOOO5, The Learning and Working Transitions RRTC). NIDILRR is a Center within the Administration for Community Living
(ACL), Department of Health and Human Services (HHS). The contents of this tip sheet do not necessarily represent the policy of MADMH, NIDILRR, ACL, or HHS and you should not assume endorsement by local or the Federal Covernment. Suggested Citation: Larkin, C. (2021). Ways to mind your mental health in challenging times: Tips for youth. Worcester, MA: University of Massachusetts Medical School, Department of Psychiatry, Implementation Science and Practice Advances Research Center (iSPARC), Transitions to Adulthood Center for Research. 